\title{
Manipulating host resistance structure reveals impact of pathogen dispersal and environmental heterogeneity on epidemics
}

\section{Penczykowski, Rachel M.}

2018-12

Penczykowski , R M , Parratt , S R , Barres , B , Sallinen , S K \& Laine , A-L 2018 , '

Manipulating host resistance structure reveals impact of pathogen dispersal and environmental heterogeneity on epidemics ' , Ecology , vol. 99 , no. 12 , pp. 2853-2863 . https://doi.org/10.1002/ecy.

http://hdl.handle.net/10138/297082

https://doi.org/10.1002/ecy.2526

publishedVersion

Downloaded from Helda, University of Helsinki institutional repository.

This is an electronic reprint of the original article.

This reprint may differ from the original in pagination and typographic detail.

Please cite the original version. 


\title{
Manipulating host resistance structure reveals impact of pathogen dispersal and environmental heterogeneity on epidemics
}

\author{
Rachel M. Penczykowski, ${ }^{1,2,5}$ Steven R. Parratt, ${ }^{1,3}$ Benoit Barrès, ${ }^{1,4}$ Suvi K. Sallinen, ${ }^{1}$ and Anna-Liisa Laine ${ }^{1}$ \\ ${ }^{1}$ Research Centre for Ecological Change, University of Helsinki, PO Box 65 (Viikinkaari 1), FI-00014 Helsinki Finland
}

\begin{abstract}
Understanding how variation in hosts, parasites, and the environment shapes patterns of disease is key to predicting ecological and evolutionary outcomes of epidemics. Yet in spatially structured populations, variation in host resistance may be spatially confounded with variation in parasite dispersal and environmental factors that affect disease processes. To tease apart these disease drivers, we paired surveys of natural epidemics with experiments manipulating spatial variation in host susceptibility to infection. We mapped epidemics of the wind-dispersed powdery mildew pathogen Podosphaera plantaginis in five populations of its plant host, Plantago lanceolata. At 15 replicate sites within each population, we deployed groups of healthy potted 'sentinel' plants from five allopatric host lines. By tracking which sentinels became infected in the field and measuring pathogen connectivity and microclimate at those sites, we could test how variation in these factors affected disease when spatial variation in host resistance and soil conditions was minimized. We found that the prevalence and severity of sentinel infection varied over small spatial scales in the field populations, largely due to heterogeneity in pathogen prevalence on wild plants and unmeasured environmental factors. Microclimate was critical for disease spread only at the onset of epidemics, where humidity increased infection risk. Sentinels were more likely to become infected than initially healthy wild plants at a given field site. However, in a follow-up laboratory inoculation study we detected no significant differences between wild and sentinel plant lines in their qualitative susceptibility to pathogen isolates from the field populations, suggesting that primarily non-genetic differences between sentinel and wild hosts drove their differential infection rates in the field. Our study leverages a multi-faceted experimental approach to disentangle important biotic and abiotic drivers of disease patterns within wild populations.
\end{abstract}

Key words: host resistance; host-parasite interaction; microclimate; parasite dispersal; plant-pathogen interaction; spatial epidemiology.

\section{INTRODUCTION}

One of the main challenges in epidemiology is to understand the causes of variability among different epidemics of the same disease (Gibson et al. 1999). Differences among epidemics may result from spatiotemporal variation in susceptible hosts, infective pathogens, and conducive environmental conditions (i.e., the three corners of the 'disease triangle'; (Stevens 1960)) at both the among-population and within-population level (Parratt et al. 2016, Penczykowski et al. 2016). Delineating how variation in each corner of the disease triangle shapes epidemics is critical for informing strategies to predict and manage diseases. However, few studies have disentangled the effects of these three sources of variation within natural populations of spatially structured hosts, where spatial variation in host resistance may be confounded with variation in dispersal of parasites and/or environmental factors that influence disease processes.

Manuscript received 23 July 2018; accepted 20 August 2018; final version received 20 September 2018. Corresponding Editor: Bitty A. Roy.

${ }^{2}$ Present address: Department of Biology, Washington University in St. Louis, St. Louis, Missouri 63130 USA.

${ }^{3}$ Present address: Department of Ecology and Evolution, Institute of Integrative Biology, University of Liverpool, Liverpool L69 7ZB UK

${ }^{4}$ Present address: Laboratoire de Lyon, Université de Lyon, ANSES, 31 Avenue Tony Garnier, F-69007 Lyon France.

${ }^{5}$ E-mail: rpenczykowski@wustl.edu
As pathogens can only establish in places where there are susceptible hosts, greater spatial heterogeneity in host resistance is expected to slow the spread of disease (Dwyer et al. 1997, Altizer et al. 2003, Altermatt and Ebert 2008, King and Lively 2012). Variation in resistance within host populations is thought to be one factor explaining the typically less devastating epidemics in natural plant populations compared to those in monoculture agricultural systems (Zhu et al. 2000, Mundt 2002, Burdon and Thrall 2014). Indeed, intraspecific crop diversification has been shown to be a highly effective means of reducing disease loads in agricultural fields (Wolfe 1985, Zhu et al. 2000, Reiss and Drinkwater 2018). Moreover, deployment of multiple cultivars of varying resistance can increase crop resistance durability by both decreasing pathogen abundance and disrupting evolutionary dynamics of the pathogen (Papaïx et al. 2017). Outside of agricultural settings, spatial heterogeneity in host resistance within populations has been documented in a broad range of host-parasite systems including vertebrate, invertebrate, and plant hosts (Thorne and Williams 1988, Baer and Schmid-Hempel 1999, Laine et al. 2011, Gibson et al. 2016). However, we still know little about how effective that variation is at limiting disease prevalence within natural populations (Alexander 2010, King and Lively 2012).

As conceptualized by the disease triangle, physical environmental variation can have a critical influence on the development of an epidemic (Stevens 1960). A wide range of 
economically and environmentally important pathogens are known to be highly sensitive to environmental factors (Altizer et al. 2006, Wolinska and King 2009). For example, many species of fungi have threshold temperatures and humidity levels for germination to occur (Truscott and Gilligan 2003). Consequently, variability in physical conditions such as temperature and rainfall may induce pronounced fluctuations in population dynamics of pathogenic fungi (Burdon 1993, Warren and Mordecai 2010). Within populations of spatially structured hosts, small-scale environmental variation can be a major driver of spatial patterns of disease (Borer et al. 2010).

In natural environments, the prevalence and severity of infection depends not only on the interplay between host susceptibility and environmental variation, but also on the locations of initial pathogen foci and pathogen dispersal ability (Ekholm et al. 2017). Proximity to initial disease foci has been shown to be a key determinant of infection risk across a wide range of pathosystems (Estep et al. 2014). Although the effect of distance on infection risk is expected to depend strongly on the mode of pathogen dispersal, even for airborne pathogens the vast majority of passively dispersing spores remain airborne only a short while and land within a few meters from their source (Ovaskainen and Laine 2006, Lannou et al. 2008, Tack et al. 2014). Consequently, pathogen transmission occurs mainly over short distances, often resulting in highly aggregated infection prevalence and spatially variable exposure to pathogens within host populations (Ovaskainen and Laine 2006, Estep et al. 2014).

Effects of host genetics and environmental variation on disease transmission and development have been repeatedly shown in laboratory and experimental garden studies (Mitchell et al. 2005, Laine 2007, Busby et al. 2014), yet their relative importance in driving natural epidemics has remained elusive. In the present study, we wanted to determine how (1) spatial variation in parasite exposure, (2) environmental factors, and (3) host genotype and condition affect infection risk and severity. To capture the natural range of variation in disease prevalence, we intensively surveyed the epidemic spread of the fungal pathogen Podosphaera plantaginis in five populations of its plant host Plantago lanceolata across the Aland archipelago (SW Finland). Throughout these populations, we deployed small groups of potted healthy 'sentinel' plants, which were host lines chosen for their generally high susceptibility to Po. plantaginis. By tracking which sentinels became infected, we were able to test effects of short-distance pathogen dispersal (i.e., proximity to infected wild plants) and environmental heterogeneity (i.e., measurements of microclimate and within-population location) on epidemiology, without confounding spatial variation in host resistance or soil conditions. We deployed two cohorts of sentinels, which allowed us to evaluate these drivers of disease both early and late in the epidemics. In addition, we tested whether sentinels had greater prevalence of infection compared to adjacent wild plants. Then, we performed a laboratory inoculation experiment on greenhouse-grown hosts from wild and sentinel lines to determine whether differences in their infection prevalence in the field were explained by differences in their laboratory-measured susceptibility.

\section{Materials And Methods}

\section{Study system}

The host plant, Plantago lanceolata L. (ribwort plantain), is a monoecious perennial rosette-forming herb (Sagar and Harper 1964). This species is host to the obligate parasite Podosphaera plantaginis (Castagne; U. Braun and S. Takamatsu), a powdery mildew fungus in the order Erysiphales. The fungus grows on the leaf surface, and only its haustoria enter the epidermis to extract plant nutrients (Bushnell 2002). Mycelia growing on the leaf surface produce chains of asexual, wind-dispersed transmission spores (conidia). Repeated cycles of clonal pathogen reproduction during summer are followed by sexual production of resting structures (chasmothecia) through either haploid selfing or outcrossing (Tollenaere and Laine 2013). The chasmothecia enable the pathogen to survive winter when the host plant has died back to rootstock, and re-initiate epidemics in spring (Tack and Laine 2014).

In the Aland archipelago, Po. plantaginis persists as a metapopulation on dry meadows and pastures (Ojanen et al. 2013) with frequent extinctions and recolonizations in the ca. $4000 \mathrm{Pl}$. lanceolata populations (Laine and Hanski 2006, Jousimo et al. 2014). The metapopulation dynamics of this host-pathogen interaction in Alland have been well studied since the year 2001 (Laine and Hanski 2006, Jousimo et al. 2014). Successful infection depends on compatibility between plant and pathogen genotypes consistent with the gene-for-gene model (Thompson and Burdon 1992). Evidence for diversity within and among host populations comes from laboratory inoculation experiments showing variation in resistance to a given set of pathogen strains among clonal plant lines under controlled conditions (Laine 2004, 2007). Hosts that are qualitatively susceptible to a given pathogen strain may still vary in their ability to mitigate its sporulation once infected (i.e., quantitative resistance). The Po. plantaginis populations in Alland are also diverse, comprised of genetically and phenotypically distinct pathogen strains (Tack et al. 2014), with a high proportion of coinfection by different multilocus genotypes (MLGs) (Tack et al. 2014, Susi et al. 2015).

\section{Focal populations}

To study drivers of powdery mildew epidemics over small spatial scales, we paired intensive weekly surveys of disease (see Epidemic surveys) with manipulations of available host genotypes (see Field experiment) in five Pl. lanceolata populations in Åland during summer 2014. These populations, (IDs: 475, 490, 3301, 9029, and 9066, Fig. 1A, Appendix S1: Table S1), were selected because they were (1) infected with Po. plantaginis in 2012, 2013, and at the beginning of July 2014, (2) large enough to meet criteria for sentinel plant placement (see Field experiment), and (3) subject to little disturbance by humans or large grazers.

\section{Epidemic surveys}

We mapped powdery mildew epidemics by surveying the five populations weekly from the onset of epidemics on 3 July 

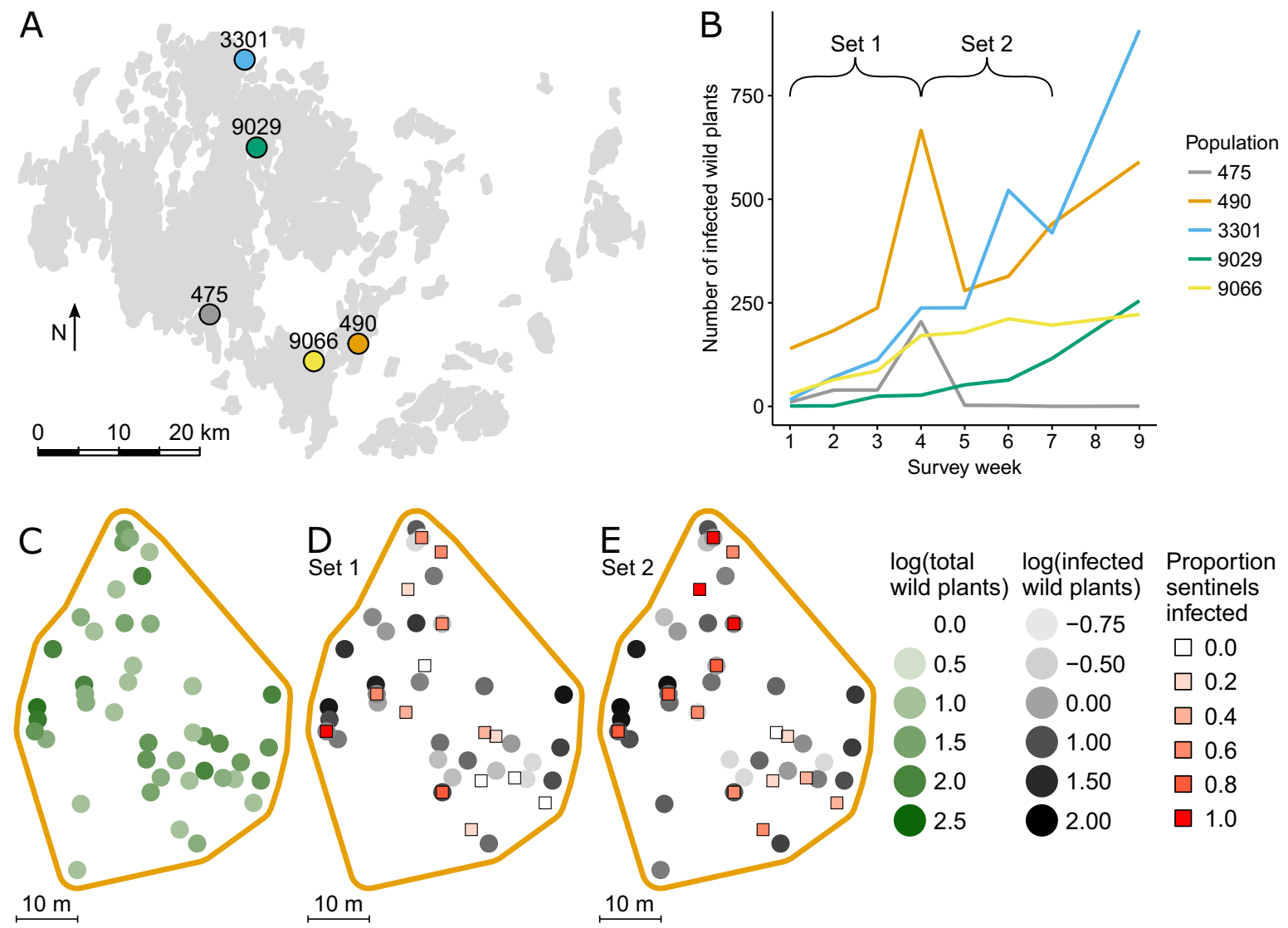

$\log ($ infected Proportion

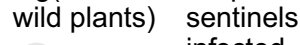

wild plants) sentinels

$-0.75 \square 0.0$
$-0.50 \quad \square 0.000$

$-0.50 \square 0.2$
0.000 .4

$0.00 \square 0.4$

1.00

$\square 0.6$

1.50

$\square 0.8$

2.00

$\square 1.0$

FIG. 1. Epidemics in the focal populations. (A) Map of the Aland Islands, Finland, showing the five focal populations. (B) Time series of infected wild host plants in each population. (C-E) Maps of host abundance and infection in population 490. (C) Maximum number of wild hosts observed in each survey circle (green shaded circles). (D-E) Mean number of infected wild hosts (gray shaded circles) and proportion of sentinel plants infected (red shaded squares) during the (D) Set 1 and (E) Set 2 exposure periods. Survey circles (radius $1.5 \mathrm{~m}$ ) are drawn to scale, but squares representing $0.15 \mathrm{~m}^{2}$ trays of sentinels are not.

to 14 August 2014 (weeks 1-7), and again when disease spread had ceased in the last week of August (week 9). On each survey date, we walked through the entire host population and visually screened for the conspicuous white mycelia and conidia of Po. plantaginis growing on Pl. lanceolata. When an infected plant was found, we marked it with a tag inserted into the ground, and recorded its coordinates using a GPS device (Transystem 737A+ receiver with AGPS function, Hsinchu, Taiwan). We then inspected the area within a $1.5 \mathrm{~m}$ radius of the marked infected plant (hereafter, 'survey circle') and recorded the percentage coverage by uninfected and infected host plants, which we converted to an estimate of number of plants assuming that 10 plants covered $1 \%$ of the survey circle. We re-surveyed the circles each week, and marked new infected plants and survey circles as infections spread, until up to 30 non-overlapping circles were marked in a given population. Beginning in week 3, we also surveyed wild plants within $1.5 \mathrm{~m}$ of trays of sentinel plants (see Field experiment). Although the centers of survey circles were at least $3 \mathrm{~m}$ apart as measured on the ground, the GPS device was only accurate to approximately $3 \mathrm{~m}$ (2DRMS 95\%); thus, some circles appear overlapping when their coordinates are plotted.

Because plants with larger surface area of leaf tissue should be more likely to encounter passively dispersing pathogen spores, we included foliar surface area as a covariate in some statistical analyses. We estimated average surface area of wild plants in each population based on measurements of 50 randomly selected plants in survey week 4 . For each plant, surface area was estimated as number of leaves $\times$ average leaf length $\times$ average leaf width.

\section{Field experiment using sentinel plants}

Simultaneous with the epidemic surveys, we performed a field experiment using sentinel plants to tease apart multiple hypothesized drivers of epidemiology. The sentinels were from five maternal lines, all allopatric to the experimental populations (Appendix S1: Table S1), and grown from seed in a greenhouse under standardized, pathogen-free conditions. These sentinel lines were chosen because they were previously shown to be susceptible to a broad array of pathogen strains (Susi and Laine 2013); we later quantified their susceptibility to strains occurring in the focal populations in 2014 (see Laboratory inoculation experiment).

We placed two consecutive cohorts of sentinels into the five focal populations, to determine if different factors affected their probability and severity of disease early vs. later in the epidemics. The Set 1 and Set 2 sentinels were planted in a greenhouse two weeks apart in May 2014, and the Set 1 sentinels were deployed into the field on 3 July 
2014. Sentinels were deployed in pots of greenhouse soil within $0.15 \mathrm{~m}^{2}$ impermeable plastic trays, rather than transplanted into the soil, to minimize their spatial variation in soil condition and directly test effects of other variables of interest. Each tray contained one sentinel plant from each of the five maternal lines, and we placed 15-16 trays into each population. Within each tray, the five sentinel plants were watered and rotated every two days to minimize differences in their pathogen exposure and micro-environmental conditions. Tray sites were selected according to these criteria: (1) adjacent to wild Plantago, (2) at least $2 \mathrm{~m}$ from another tray and from the nearest infected plant on 3 July 2014, and (3) approximately evenly distributed across the host population. The Set 2 sentinels were later placed at those same sites.

Both sets of sentinel plants were left in the field for $20 \mathrm{~d}$ of exposure to pathogens (Set 1: 3-23 July 2014, Set 2: 23 July-13 August 2014), allowing sufficient time for contact with Po. plantaginis spores while limiting the release of new spores from infected sentinels into the wild populations. After the exposure period, sentinels were collected into individual spore-proof pollination bags (PBS International, type 2D.1-1W, no. 0000791) to avoid cross-contamination, and transported to the Kumpula Botanical Gardens (Helsinki, Finland). There, the covered plants were placed outside for an additional two weeks to allow infections to continue developing. We assessed infection status of every leaf on each plant at both one and two weeks following the exposure period, and used the maximum numbers of total and infected leaves in our analyses. We estimated the surface area of each sentinel as described above for wild plants (see Epidemic surveys). In addition, we collected infected leaf samples for the follow-up inoculation experiment (see Laboratory inoculation experiment).

\section{Within-population connectivity to mildew}

From the epidemic survey data, we calculated the connectivity of sentinels at each tray site to all infected wild plants surveyed within the same population. This within-population connectivity metric $(S)$ takes into account the distance (d) of a given tray site ( $i$ ) to the center of each survey circle $(j)$ as well as the cumulative intensity of powdery mildew infection ( $p$, defined as $\%$ host coverage $\times \%$ infected hosts, summed over the Set 1 or Set 2 exposure period) of wild plants in each survey circle $j$. We defined within-population connectivity to mildew, $S$, as

$$
S_{i}=\sum p_{j} \exp \left(-\alpha d_{i j}\right)
$$

where the probability of pathogen spore dispersal from each site $j$ to plants at site $i$ declines exponentially with distance between sites, according to an average dispersal distance $(1 / \alpha)$ of $2 \mathrm{~m}$. Previous work in this system has shown that most spores fall within $1 \mathrm{~m}$ of infected plants, and dispersal $>2 \mathrm{~m}$ is rare (Ovaskainen and Laine 2006, Tack et al. 2014). Fitting a single dispersal kernel to wind-dispersed species is always a simplification (Shaw 1995), but this approximation has been show to capture within-population dynamics (Ovaskainen and Laine 2006). Here we tried several different values of $\alpha$, and our results remained robust over dispersal distances ranging from $<0.5 \mathrm{~m}$ to more than $10 \mathrm{~m}$.

\section{Microclimate}

To evaluate effects of microclimate on sentinel plant infection risk, we used data loggers (EL-USB-2, Lascar Electronics) to record hourly measurements of air temperature and relative humidity at $15 \mathrm{~cm}$ above the ground next to 14 trays of sentinels per population. We installed each logger on a rebar stake with a bucket shield against direct rain and sun. As all loggers had the same type of bucket shield, any effect on microclimate would be systematic. For each logger, we computed daytime (08:00-21:00) averages of temperature and humidity over the two sentinel exposure periods, as daytime climatic conditions are critical for the development and transmission of powdery mildew infection (Nicot et al. 2002, Glawe 2008). One logger from population 490 malfunctioned and was excluded from the analyses. To estimate temperature and humidity at sites without loggers, we used kriging ('autoKrige' in R package 'automap'; (Hiemstra et al. 2009, R Core Team 2015)) to interpolate values within the region bounded by the coordinates of the loggers, and extracted the interpolated values at locations of tray sites lacking loggers using a buffer of $2 \mathrm{~m}$ (package 'raster'; [Hijmans 2015]). In analyses, we used measured values at sites with loggers and interpolated values at sites lacking loggers (Appendix S1: Figs. S1, S2). Air temperature and relative humidity were significantly correlated across sites (Set 1: $r=-0.91$, $P<0.0001$; Set 2: $r=-0.87, P<0.0001$ ). We used relative humidity (not temperature) as an explanatory variable in the analyses because powdery mildews such as Po. plantaginis are particularly sensitive to moisture in the air, requiring moderate relative humidity - but not free water - for successful germination and transmission (Glawe 2008).

\section{Laboratory inoculation experiment}

Following the field experiment, we performed a laboratory experiment to compare the susceptibility of sentinel and wild plants to pathogen isolates from the focal populations. As described below, we grew sentinel and wild plant lines from seed in the greenhouse, inoculated them with pathogen isolates from the five populations, and measured infection outcomes qualitatively (infected or not) and quantitatively (amount of pathogen sporulation).

Plant lines. - In mid-August 2014, we collected seeds to establish maternal lines derived from five infected and five uninfected $\mathrm{Pl}$. lanceolata individuals from each of the five populations. Each plant line came from a single seed head from a mother plant, and we selected mother plants from locations spread across each host population in an effort to capture the variation in plant genetic background within each population. Seeds from these wild plants, as well as from the five sentinel plant lines (Appendix S1: Table S1) were sown in $9 \times 9 \mathrm{~cm}$ flower pots in a mixture of $30 \%$ sand, $70 \%$ potting soil. Plants were maintained in the greenhouse at $+20^{\circ} \mathrm{C}$ until inoculation.

Pathogen isolates. - We collected and purified isolates of Po. plantaginis from wild Pl. lanceolata in the focal populations, and from the Set 2 sentinels. Infected leaves were detached using forceps (sanitized with DNA-Away, Molecular 
Bio Products) and placed into $9 \mathrm{~cm}$ Petri dishes containing moist filter paper. We collected an infected leaf from at least 10 wild plants per population at the end of the 2014 epidemics; however, no wild infected plants were found in population 475 at that time. Several pathogen isolates died during purification and maintenance, hence 7-10 isolates from each of the four wild plant population were used in the experiment. Pathogen isolates were also collected from sentinel plants from all five populations (3-7 viable isolates each). To ensure that each pathogen isolate was a single MLG, we purified the isolates through three single-colony transfers of spores onto detached, greenhouse-grown leaves (Nicot et al. 2002). Inoculated leaves were maintained on moist filter paper in Petri dishes in a growth chamber under standard conditions of $21^{\circ} \mathrm{C}\left( \pm 2^{\circ} \mathrm{C}\right)$ and $16 \mathrm{~L}: 8 \mathrm{D}$ photoperiod. We amplified the fungal isolates through 2-3 rounds of inoculations to generate spores for the experiment described below.

Inoculations and scoring of infection.-We inoculated each pathogen isolate onto 10 sympatric wild plant lines (five from seeds of infected wild plants, five from seeds of uninfected wild plants) and the set of five allopatric sentinel lines. All inoculations were performed in November-December 2014. Healthy leaves from the greenhouse-grown plant lines were detached and placed on moist filter paper in Petri dishes, where they were exposed to spores of the purified pathogen strains following methods in Laine (2007). Each Petri dish contained four leaf pieces - two from wild plant lines, two from sentinel lines - and each dish was inoculated with a single pathogen isolate. Because the sentinel lines were previously found to be susceptible to a variety of pathogen strains (Susi and Laine 2013), having two sentinel lines on each dish acted as a quality control for the inoculations. To standardize the dose of pathogen spores, we used a fine paint brush to pick up conidial spores from lesions of the same age and size (approximately $1.0 \mathrm{~cm}$ diameter) and brush them over the entire surface of the healthy leaf. We maintained the inoculated leaves in a growth chamber under standard conditions.

We examined the leaves under a dissecting microscope (Nikon NMZ800) at $12 \mathrm{~d}$ post-inoculation, and scored the presence (1) and absence (0) of conidial spores. The amount of sporulation was scored on a modified version of the scale introduced by Bevan et al. (1993), where $0=$ no infection, $1=$ only mycelia, 2 = mycelia and conidia, with lesions visible only under a microscope, $3=$ lesions visible with the naked eye, and sparse sporulation, $4=$ lesions of under $0.5 \mathrm{~cm}$ diameter with profuse sporulation, and $5=$ lesions of over $0.5 \mathrm{~cm}$ diameter with profuse sporulation. We repeated any inoculations that failed due to death or contamination of the leaf material, or when none of the leaves on the plate became infected. We analyzed the maximum infection response of replicated host-pathogen pairings.

\section{Statistical analyses}

Statistical analyses were performed in R ( R Core Team 2015). In the epidemic surveys, we tested whether maximum infection prevalence of wild hosts in a survey circle was explained by the log-transformed maximum total number of wild hosts in that survey circle. We did so using a generalized linear mixed effects model (GLMM; package 'lme4') with binomial distribution of errors and logit link function, and a random effect of population.

In the field experiment, we first tested which factors influenced the proportion of sentinel plants becoming infected at each site in Sets 1 and 2 by fitting generalized additive mixed effects models (GAMMs; package 'gamm4') with binomial distributions of errors and logit link functions. We included log-transformed connectivity to infected wild plants within the population $(\log (S))$, mean daytime relative humidity, log-transformed sentinel host surface area, and all interactions as fixed effects. These variables were all standardized as $z$-scores $\left(\left(x_{i}-\mu_{x}\right) / \sigma_{x}\right)$. We also ran versions of the models with total wild host density in the survey circle as a covariate, and found no significant effect of that term on sentinel infection risk. However, we excluded the total wild host density term from the final models due to strong collinearity with connectivity to disease, $S$ (Set $1: r=0.26, P<0.0001$, Set 2: $r=0.27, P<0.0001)$. The GAMM framework allowed us to test for spatial dependence not already accounted for by other explanatory variables by fitting thin plate regression splines (smoothing basis = "ts", basis dimension $=10$ ) of longitude and latitude centered for each population. If the effect of the smoothed spatial coordinates was not significant in any population, we fit a GLMM instead. We included population, tray site (nested within population), and sentinel plant maternal line as crossed random effects. We also tested which factors determined the severity of infection on sentinels (proportion of leaves infected, for infected plants only). These models were structured as for infection risk, but included an additional random effect of sentinel plant individual (uniquely coded). Minimum adequate models were derived from saturated models through stepwise simplification (removal of interaction terms only) and selection based on likelihood ratio tests $\left(\chi^{2}\right.$ distributed test statistic) of nested models (Crawley 2007). The effect of significant independent variables was derived from analysis of the minimum adequate model with the 'car' package for GLMMs (Wald $\chi^{2}$ ) and model simplification for GAMMs (LRT $\left.\chi^{2}\right)$.

In the field experiment, the Set 1 sentinel plants were placed at sites with only healthy wild plants in the beginning of epidemics (i.e., at least $2 \mathrm{~m}$ from initial disease foci), so we could compare the proportion of sentinel and wild plants that acquired infections at each site during the Set 1 exposure period. To test if a greater proportion of sentinels became infected than wild plants at the same sites (i.e., within $1.5 \mathrm{~m}$ of trays), we used a GLMM with binomial distribution of errors and logit link function. As infections on sentinels were scored two weeks following their removal from the field populations, the response variable for wild plants was calculated as the maximum proportion infected at a given tray site over the same total time period (i.e., weeks $1-6$ of the epidemic). Plant type (sentinel or wild) was coded as a fixed effect, and population and site (nested within population) were random effects.

For the laboratory inoculation experiment, we analyzed the qualitative (infected or not) and quantitative (Bevan's score index of sporulation) infection response of each host line-pathogen isolate pair. The qualitative infection responses were modeled using a GLMM (binomial distribution, logit 
link), and Bevan's scores were modeled using a cumulative link mixed model (CLMM; package 'ordinal') appropriate for this ordered categorical response variable (Christensen 2015). In the analysis of quantitative infection response, only leaves with Bevan's score $\geq 1$ were included, to test how well mildew could grow if it was able to germinate. In both analyses, we tested the fixed effects of plant line type (sentinel, wild infected, or wild uninfected maternal lines), pathogen population, and their interaction, with plant line and pathogen isolate included as uniquely coded random effects. We performed a second set of analyses with the offspring of wild infected and wild uninfected plants grouped together as one level of plant line type (i.e., a comparison of allopatric vs. sympatric plant lines). In preliminary analyses, pathogen isolate origin (whether a pathogen isolate from a given population was collected from a wild or a sentinel plant) and its possible interactions with other variables were included as fixed effects and found to be highly non-significant in all models; thus, this variable was excluded from the final analyses. In the CLMM, significance of fixed effects was assessed with likelihood ratio tests, and we used the package 'lsmeans' to perform post-hoc pairwise comparisons between plant line types for each population (Tukey's HSD tests).

\section{RESUlts}

\section{Epidemic surveys}

The maximum number of resident $P l$. lanceolata individuals observed to be infected with $P$ o. plantaginis varied among the five focal populations, ranging from approximately 200 to over 900 plants (Fig. 1B). While the epidemics continued to grow over the course of the study period in four of the populations, the epidemic in population 475 peaked in week 4 before ending due to drought (Fig. 1B). Within each population, the number and proportion of infected wild plants varied greatly over the scale of a few meters (Fig. 1C-E; Appendix S1: Fig. S3). There was a significant positive relationship between the total density and infection prevalence of wild plants in the survey circles $\left(\chi^{2}=13.89\right.$, df $\left.=1, P=0.0002\right)$.

\section{Field experiment}

Infection prevalence varied greatly among trays of sentinels in both Set 1 and 2 (Fig. 1C-E and Appendix S1: Fig. S3; Table 1 and Appendix S1: Table S2). This spatial heterogeneity in infection risk was largely explained by connectivity to wild infected plants $(S)$, where sentinels located at sites with higher local disease prevalence were more likely to become infected (Set 1: $\chi^{2}=20.87, \mathrm{df}=1, P<0.0001$, Fig. 2A; Set 2: $\chi^{2}=12.97, \mathrm{df}=1, P=0.0003$, Fig. $\left.2 \mathrm{~B}\right)$. The probability of infection for the Set 1 sentinel plants was further increased by greater daytime relative humidity $\left(\chi^{2}=5.09, \mathrm{df}=1\right.$, $P=0.024$, Fig. 2C), while host plant surface area was not a significant covariate $\left(\chi^{2}=1.20, \mathrm{df}=1, P=0.27\right.$, Fig. 2E). In Set 2, sentinel plant infection risk was not significantly affected by relative humidity $\left(\chi^{2}=0.52\right.$, df $=1, P=0.47$, Fig. 2D), but did significantly increase with plant surface area $\left(\chi^{2}=13.94, \mathrm{df}=1, P=0.0002\right.$, Fig. $\left.2 \mathrm{~F}\right)$. The standard deviation in the random effect of tray site was similar in magnitude to the largest estimated fixed effect in both Set 1
TABLE 1. Factors explaining the probability of infection for sentinel plants in the field experiment, analyzed using a GLMM in Set 1 and GAMM in Set 2 (binomial distribution, logit link).

\begin{tabular}{lrrrl}
\hline \hline Fixed effect & Estimate & SE & Wald $z$ & $P$-value \\
\hline Set 1 & & & & \\
$\quad$ Intercept & -1.08 & 0.21 & -5.15 & $<\mathbf{0 . 0 0 0 1}$ \\
$\quad$ Log(Connectivity to & 0.89 & 0.19 & 4.57 & $<\mathbf{0 . 0 0 0 1}$ \\
$\quad$ pathogen, $S$ ) & & & & \\
$\quad$ Log(Host surface area) & 0.15 & 0.14 & 1.09 & 0.27 \\
$\quad$ Humidity & 0.38 & 0.17 & 2.26 & $\mathbf{0 . 0 2 4}$ \\
$\quad$ Set 2 & & & & \\
$\quad$ Intercept & -0.72 & 0.17 & -4.17 & $<\mathbf{0 . 0 0 0 1}$ \\
$\quad$ Log(Connectivity to & 0.85 & 0.21 & 4.07 & $<\mathbf{0 . 0 0 0 1}$ \\
$\quad$ pathogen, $S$ ) & & & & \\
$\quad$ Log(Host surface area) & 0.51 & 0.13 & 3.85 & $\mathbf{0 . 0 0 0 1 2}$ \\
$\quad$ Humidity & 0.12 & 0.16 & 0.74 & 0.46 \\
\hline
\end{tabular}

Notes: Estimates and standard errors (SE) for the intercept and slope parameters are given for fixed effects. Significant $P$-values are indicated in bold. Non-significant interaction terms dropped through model comparison are reported in Appendix S1: Table S2.

$(\sigma=0.85)$ and Set $2(\sigma=0.84)$, and there was no discernible among-population variation in either Set 1 or Set 2 $(\sigma=0.00)$. The estimated standard deviation among maternal lines was relatively small in Set $1(\sigma=0.24)$ and negligible $(\sigma=0.00)$ in Set 2 (Appendix S1: Fig. S4A-B). Our analysis also accounted for significant small-scale spatial autocorrelation in the proportion of sentinels infected in two populations in Set 2 (smoothed spatial coordinates term for population 490: estimated [est.] $\mathrm{df}=1.38$, reference [ref.] $\mathrm{df}=9$, $\chi^{2}=9.20, P=0.0022$; for population 3301 : est. $\mathrm{df}=1.47$, ref. df $\left.=9, \chi^{2}=7.59, P=0.0064\right)$.

Among infected sentinel plants, the proportion of leaves infected varied within populations, and greatly increased with connectivity to mildew during both exposure periods (Set 1: $\chi^{2}=11.19$, df $=1, P=0.0008$, Fig. 2G; Set 2: $\chi^{2}=11.65, \quad \mathrm{df}=1, \quad P=0.0006, \quad$ Fig. $2 \mathrm{H} ;$ Table 2 and Appendix S1: Table S2). Infection severity was not significantly related to daytime relative humidity at the site (Set 1: $\chi^{2}=1.71, \quad \mathrm{df}=1, \quad P=0.19 ; \quad$ Set $2: \quad \chi^{2}=1.81, \quad \mathrm{df}=1$, $P=0.18)$, or to plant surface area (Set 1: $\chi^{2}=0.02, \mathrm{df}=1$, $P=0.88$; Set $2: \chi^{2}=0.13, \mathrm{df}=1, P=0.72$ ). Relative to the magnitude of the estimated fixed effects, there was large standard deviation among tray sites (Set 1: $\sigma=0.72$, Set 2: $\sigma=1.00$ ) and among individuals (Set 1: $\sigma=1.01$, Set 2: $\sigma=0.92$ ). In Set 1 , there was additional variation in infection severity among populations $(\sigma=0.41)$ and among maternal lines ( $\sigma=0.30$, Appendix S1: Fig. S4C), as well as within-population spatial autocorrelation in sentinel disease severity in population 3301 (est. $\mathrm{df}=1.29$, ref. $\mathrm{df}=9$, $\left.\chi^{2}=4.22, P=0.042\right)$. In Set 2 , there was approximately no variation in the random effect of population $(\sigma=0.00)$ or maternal line $(\sigma=0.00$, Appendix S1: Fig. S4D).

At a given site within a population, sentinel plants were more likely to become infected than wild plants during the Set 1 exposure period $\left(\chi^{2}=124.58\right.$, df $=1, P<0.0001$; Fig. 3A and Appendix S1: Fig. S5; Table S3). While wild plants outnumbered sentinels at each tray site (Appendix S1: Fig. S6A), individual sentinels had on average 15 times greater foliar surface area than wild plants in these populations (Appendix S1: Fig. S6B). 


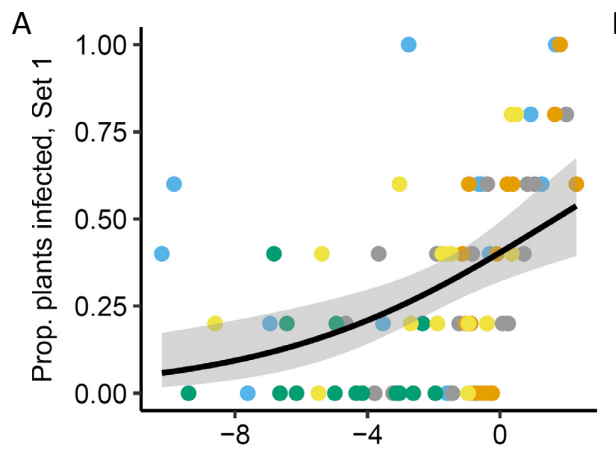

$\log ($ connectivity to infection, $S)$, Set 1

C

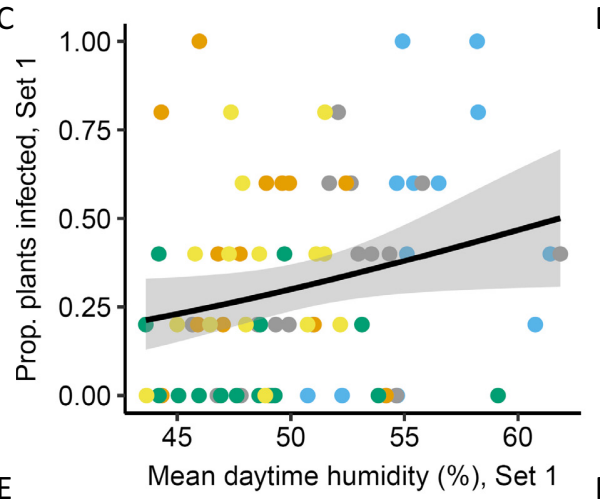

$\mathrm{E}$

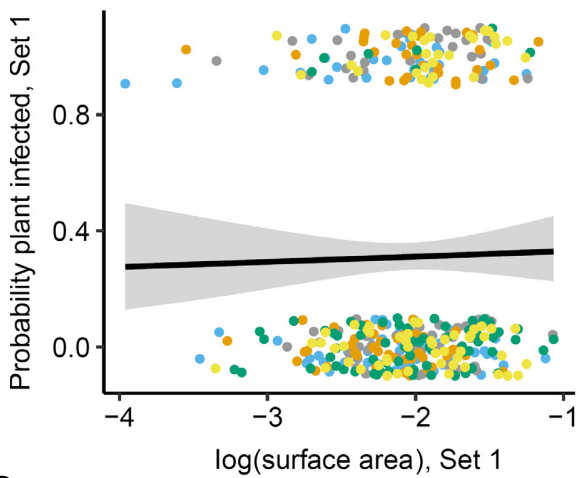

G

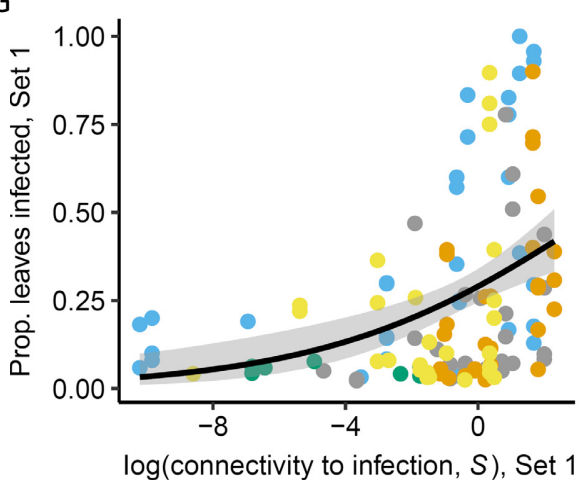

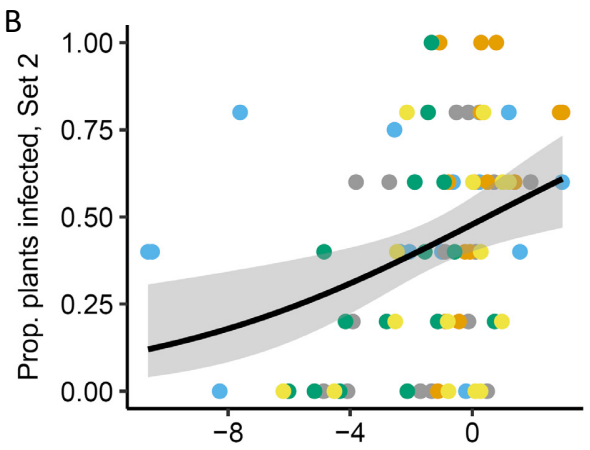

$\log ($ connectivity to infection, $S$ ), Set 2
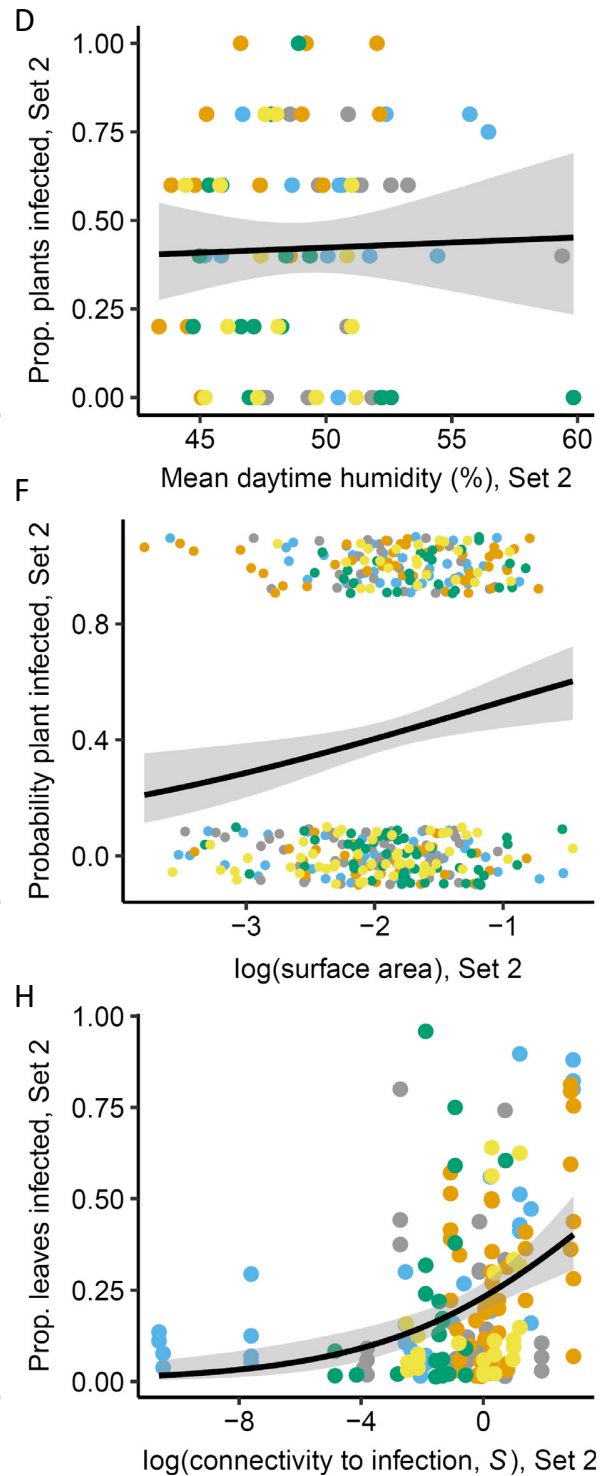

FIG. 2. Factors explaining infection risk and severity for sentinel plants. Points are trays of sentinel plants in A-D and individual plants in $\mathrm{E}-\mathrm{H}$, color-coded by population as in Fig. 1. (A, B) In both Sets 1 and 2, a greater proportion of sentinels were infected at sites that were more highly connected to infected wild plants. (C) Sentinel infection risk increased with daytime relative humidity during Set 1, but (D) there was no effect of humidity during Set 2. (E) Host size did not predict infection risk in Set 1, but (F) larger sentinel plants had greater infection risk in Set 2. $(\mathrm{G}, \mathrm{H})$ For infected sentinels, the proportion of leaves infected increased with connectivity to infected wild plants. Grey envelopes show the $95 \% \mathrm{CI}$ around a quasibinomial (A-D, G, H) or binomial (E, F) GLM fit across all points. 
TABLE 2. Factors explaining the severity of infection (proportion of leaves infected) for sentinel plants in the field experiment, analyzed using a GAMM in Set 1 and GLMM in Set 2 (binomial distribution, logit link)

\begin{tabular}{lrrrc}
\hline \hline Fixed effect & Estimate & SE & Wald $z$ & $P$-value \\
\hline Set 1 & & & & \\
$\quad$ Intercept & -2.01 & 0.30 & -6.71 & $<\mathbf{0 . 0 0 0 1}$ \\
$\quad$ Log(Connectivity to & 0.78 & 0.21 & 3.78 & $\mathbf{0 . 0 0 0 1 6}$ \\
$\quad$ pathogen, $S$ ) & & & & \\
$\quad$ Log(Host surface area) & -0.02 & 0.12 & -0.15 & 0.88 \\
$\quad$ Humidity & 0.34 & 0.20 & 1.71 & 0.087 \\
Set 2 & & & & \\
$\quad$ Intercept & -1.94 & 0.17 & -11.16 & $<\mathbf{0 . 0 0 0 1}$ \\
$\quad$ Log(Connectivity to & 0.66 & 0.19 & 3.41 & $\mathbf{0 . 0 0 0 6 4}$ \\
$\quad$ pathogen, $S$ ) & & & & \\
$\quad$ Log(Host surface area) & -0.04 & 0.11 & -0.36 & 0.72 \\
$\quad$ Humidity & 0.23 & 0.17 & 1.35 & 0.18 \\
\hline
\end{tabular}

Notes: Estimates and standard errors (SE) for the intercept and slope parameters are given for fixed effects. Significant $P$-values are indicated in bold. Non-significant interaction terms dropped through model comparison are reported in Appendix S1: Table S2.

\section{Laboratory inoculation experiment}

Under controlled conditions, sentinel plant lines were highly qualitatively susceptible to pathogen isolates from each population, and the offspring of wild infected or uninfected plants were not significantly less susceptible than sentinels (plant line type: $\chi^{2}=2.00, \mathrm{df}=2, P=0.37$; Fig. $3 \mathrm{~B}$; Appendix S1: Table S4). There was no significant interaction between plant line type and population $\left(\chi^{2}=4.49\right.$, $\left.\mathrm{df}=8, P=0.81\right)$, nor significant main effect of population $\left(\chi^{2}=8.37, \mathrm{df}=4\right.$, $P=0.079$ ). Relative to the magnitude of the fixed effects, there was large variation in qualitative susceptibility among maternal lines, and less variation in the random effect of pathogen isolate (Appendix S1: Table S4). Results were qualitatively similar when the two groups of wild plant lines were pooled (plant line type $\times$ population: $\chi^{2}=2.91, \mathrm{df}=4$, $P=0.57$; plant line type: $\chi^{2}=1.57$, df $=1, P=0.21$, population: $\chi^{2}=8.31$, df $=4, P=0.081$; Appendix S1: Table S5).

The amount of sporulation on infected leaves (i.e., quantitative susceptibility) depended on the interaction between plant line type and pathogen population $\left(\chi^{2}=19.23\right.$, df $=8$, $P=0.014$; Fig. 3C; Appendix S1: Table S4). Post-hoc tests revealed significantly greater sporulation on sentinels than wild infected plant lines in population $3301(P=0.0001)$ and greater sporulation on sentinels than wild uninfected plant lines in population $9066(P=0.013)$. There were approximately equal amounts of variation in the random effects of maternal line and pathogen isolate (Appendix S1: Table S4). A marginally significant interaction between plant line type and pathogen population remained when we repeated the analysis with the two groups of wild plant line types combined $\left(\chi^{2}=9.33\right.$, df $=4, P=0.053$; Appendix S1: Table S5).

\section{Discussion}

Our field and laboratory experiments together allowed us to disentangle genetic and environmental drivers of epidemiology over small spatial scales within wild populations. In the field experiment, infection risk and severity were greatly increased for sentinels in closer proximity to infected wild plants (i.e., with greater connectivity to mildew). Humidity further increased infection risk at the onset of epidemics, and there were additional effects of environmental variation linked to the precise location of sentinels within the population. Sentinels were more likely to become infected than initially healthy wild plants at a given site within the field populations. However, the follow-up laboratory inoculation study found no evidence for genetic differences in qualitative susceptibility between the sentinels and wild plant lines, and only two pathogen populations had greater sporulation success on the allopatric sentinels compared to groups of sympatric wild plant lines.

Our field experiment results are consistent with a major role of short-distance pathogen dispersal from infection foci in driving aggregated distributions of disease within populations (Ovaskainen and Laine 2006, Lannou et al. 2008, Estep et al. 2014, Tack et al. 2014). Although occasional long-distance spore dispersal is vital to persistence of disease in the metapopulation, the vast majority of powdery mildew spores land in the immediate vicinity of their original host (Laine and Hanski 2006, Tack et al. 2014). Thus, factors that determine initial disease foci - including connectivity to other populations, susceptibility to arriving pathogen strains, pathogen abundance in the preceding season, and pathogen overwintering success - can strongly shape spatial patterns of disease prevalence within populations (Ovaskainen and Laine 2006). Moreover, infection prevalence of wild hosts generally increased with total host density at a fine spatial scale (i.e., in our $1.5 \mathrm{~m}$ radius survey circles), as predicted for density-dependent disease transmission (Anderson and May 1978). Therefore, spatial variation in total host density and locations of initial disease foci should together be key drivers of disease prevalence patterns within populations. We also found that sentinels at sites with high local disease prevalence in wild plants were more heavily infected. This could indicate repeated allo-infection of sentinels from nearby wild infected plants during the 20 -day exposure periods, or that environmental factors promoting infection in wild plants also increased disease severity in sentinels, or both.

In addition to any environmental drivers of disease that were spatially correlated with infection prevalence of wild plants (and thus confounded with connectivity to disease), we detected a positive effect of humidity on sentinel infection risk at the onset of epidemics. Later in the epidemics, we detected no significant effect of humidity on disease risk, despite similar mean and variation in relative humidity across all sentinel sites during the Set 1 and 2 exposure periods (mean $\pm \mathrm{SD}$ in Set 1: $50.5 \pm 4.4 \%$, Set 2: $49.1 \pm 3.3 \%$ ). Both early and late in the epidemics, there were additional effects of unmeasured environmental heterogeneity on sentinel infection risk and severity, evidenced as substantial variation between tray sites and residual spatial autocorrelation within populations. These spatial effects could reflect, for example, heterogeneity in wind exposure or in the height of surrounding vegetation, which would affect the likelihood of spore arrival (Calonnec et al. 2013). Spatial variation in infection risk and severity could also stem from interactions between environmental factors and the genotype of the host and/or parasite (e.g., $G_{\text {host }} \times \mathrm{E}$ 


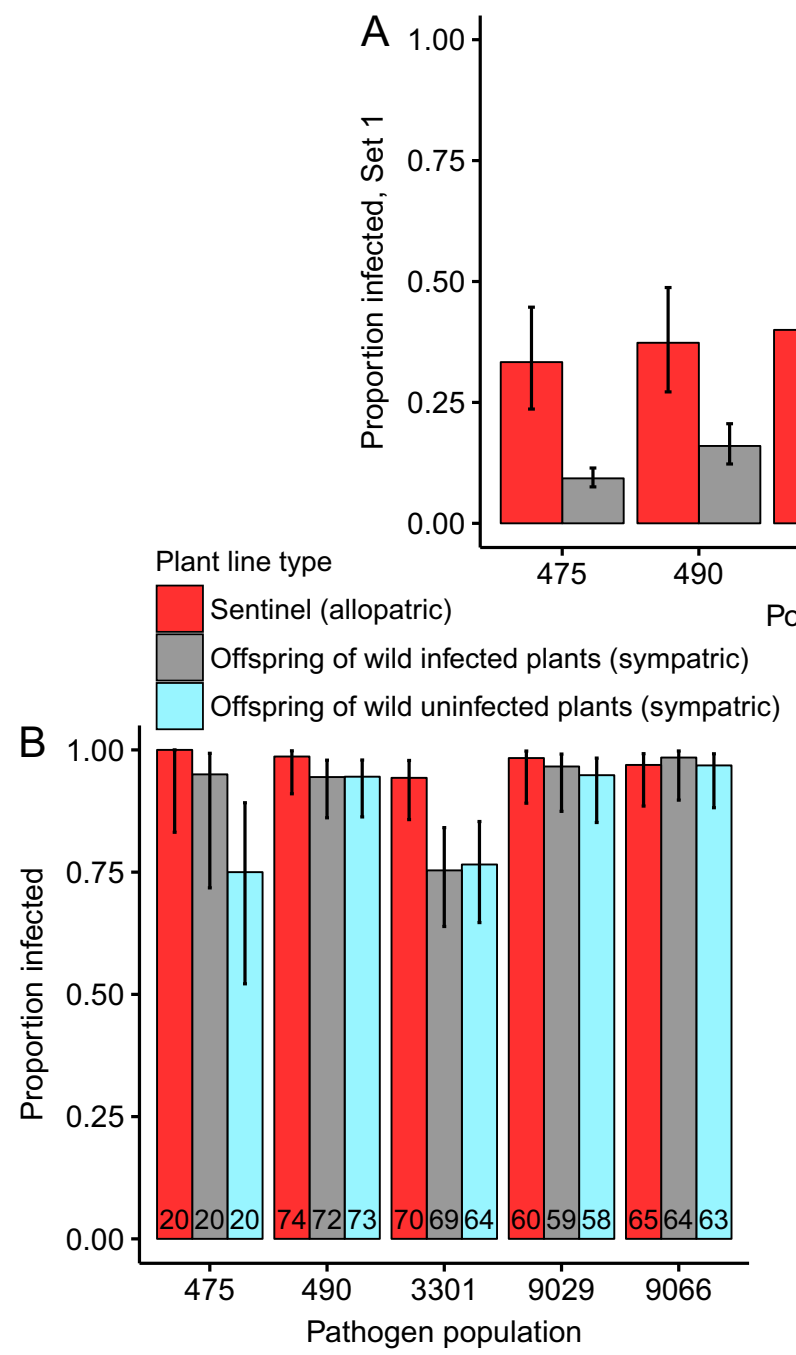

\author{
Plant type

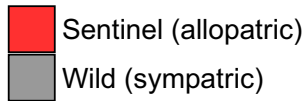 \\ Wild (sympatric)
}

FIG. 3. Susceptibility of sentinel and wild plants. (A) In the field, sentinels were more likely to become infected than adjacent wild plants during the Set 1 exposure period. In the laboratory, (B) there was no significant difference in infectivity of a population's mildew isolates when inoculated onto allopatric sentinel lines vs. offspring of sympatric wild plants, but $(\mathrm{C})$ in two populations, qualitatively infective mildew isolates were more prolific at sporulating on sentinel than wild plant lines. Significant differences from post-hoc tests are denoted with asterisks: $P<0.05(*)$, $P=0.0001(* * *)$. In B and C, the number of plant line-mildew isolate pairs tested is given at the base of each bar. Error bars show $95 \%$ CIs.

(Mitchell et al. 2005), $\mathrm{G}_{\text {parasite }} \times \mathrm{E}$ (Vale et al. 2008), or $\mathrm{G}_{\text {host }} \times \mathrm{G}_{\text {parasite }} \times$ E interactions (Wolinska and King 2009)).

The lack of significant differences in qualitative susceptibility between greenhouse-grown wild and sentinel plant lines in the controlled inoculation experiment suggests that non-genetic differences drove the greater infection rate of sentinels than neighboring wild plants in the field. As the purpose of the sentinels was to isolate effects of spatial variation in certain environmental factors (e.g., connectivity to infection foci and relative humidity), we standardized other aspects of their condition and micro-environment. This design resulted in many non-genetic differences between sentinel and wild plants in the field, including that sentinels were planted from seed in a greenhouse (vs. wild plants that may have been several years old), maintained in potting soil and watered regularly (vs. wild plants rooted in the local soil environment), and grew larger than the wild plants. While we detected no significant effect of plant size on Set 1 sentinel infection risk, the mean difference between foliar surface area of wild and sentinel plants was more than two times the standard deviation in foliar surface area among sentinels. Thus, it is likely that sentinels encountered more parasite spores than wild plants due to their larger surface area, as has been documented across diverse host-parasite systems (Seppälä et al. 2011, Penczykowski et al. 2014). Hosts that are larger or in better condition may also provide better resources for development of infection (Smith et al. 2005, Penczykowski et al. 2014). At the same time, genetic differences between sentinels and wild plants may still have played a role in their unequal infection rates, including through differences in pathogen sporulation that we detected for two populations in the inoculation experiment. It is also possible that the 10 wild plant lines sampled from each population did not adequately represent the diversity of resistance phenotypes in those populations, and that the wild plants surrounding trays of sentinels were on average more resistant than the lines used in our inoculation experiment. Ultimately, the greater infection prevalence on 
sentinels in the field - regardless of the exact combination of genetic and non-genetic factors responsible - indicates that pathogen spores were not as limiting as the spatial distribution of infected wild plants might suggest. That is, adding large, highly susceptible hosts to the populations yielded more disease than was observed in the resident wild hosts.

Although the interplay of host susceptibility and environmental conditions in shaping epidemiology has long been recognized by plant pathologists (Jones et al. 1926, Stevens 1960), modern conventional agriculture minimizes variation in genetic and environmental factors within populations. That is, many crops are bred for disease resistance, and then planted as monocultures of uniform density across homogenously managed landscapes (Zhan et al. 2015). While such practices may be effective at mitigating disease and increasing yield in the short term, pathogens typically adapt to overcome host resistance, resulting in boom-and-bust dynamics of each initially resistant host cultivar (Johnson 1961). Because wild plant populations are usually not devastated by disease outbreaks, they are a source of inspiration for alternative, sustainable disease control strategies (Zhan et al. 2015). Understanding how spatial variation in host resistance, pathogen dispersal, and the environment together shape epidemiology in patches of wild vegetation is critical for determining the potential efficacy of incorporating greater genetic or environmental heterogeneity into agricultural fields (Burdon et al. 2014). Such understanding, however, requires experiments teasing apart these potentially confounded sources of variation. Our study illustrates how an approach integrating observations of natural epidemics, manipulative field experiments, and controlled inoculation assays can help resolve how fine scale spatial variation in different corners of the disease triangle drives epidemiology in wild populations of spatially structured hosts.

\section{ACKNOWLEDGMENTS}

We thank Krista Raveala for help in the greenhouse, Pauliina Hyttinen, Sara Neggazi, Hanna Parri, and Jackson Potter for help in the field, and Guillaume Blanchet and Eric Pedersen for statistical advice. We are grateful to the Nåtö Biological Station and Kumpula Botanical Gardens for housing people and plants, respectively, during this project. Two anonymous reviewers provided helpful comments on earlier drafts of the manuscript. This work was supported by funding from the Academy of Finland (Grant Nos. 284601 and 136393) and European Research Council (PATHEVOL 400820; RESISTANCE 4100097) to A. L. Laine.

\section{Literature Cited}

Alexander, H. M. 2010. Disease in natural plant populations, communities, and ecosystems: insights into ecological and evolutionary processes. Plant Disease 94:492-503.

Altermatt, F., and D. Ebert. 2008. Genetic diversity of Daphnia magna populations enhances resistance to parasites. Ecology Letters 11:918-928.

Altizer, S., D. Harvell, and E. Friedle. 2003. Rapid evolutionary dynamics and disease threats to biodiversity. Trends in Ecology \& Evolution 18:589-596.

Altizer, S., A. Dobson, P. Hosseini, P. Hudson, M. Pascual, and P. Rohani. 2006. Seasonality and the dynamics of infectious diseases. Ecology Letters 9:467-484.

Anderson, R. M., and R. M. May. 1978. Regulation and stability of host-parasite population interactions. 1. Regulatory processes. Journal of Animal Ecology 47:219-247.
Baer, B., and P. Schmid-Hempel. 1999. Experimental variation in polyandry affects parasite loads and fitness in a bumble-bee. Nature 397:151-154.

Bevan, J. R., I. R. Crute, and D. D. Clarke. 1993. Diversity and variation in expression of resistance to Erysiphe fischeri in Senecio vulgaris. Plant Pathology 42:647-653.

Borer, E. T., E. W. Seabloom, C. E. Mitchell, and A. G. Power. 2010. Local context drives infection of grasses by vector-borne generalist viruses. Ecology Letters 13:810-818.

Burdon, J. J. 1993. The structure of pathogen populations in natural plant communities. Annual Review of Phytopathology 31:305323.

Burdon, J. J., and P. H. Thrall. 2014. What have we learned from studies of wild plant-pathogen associations?-the dynamic interplay of time, space and life-history. European Journal of Plant Pathology 138:417-429.

Burdon, J. J., L. G. Barrett, G. Rebetzke, and P. H. Thrall. 2014. Guiding deployment of resistance in cereals using evolutionary principles. Evolutionary Applications 7:609-624.

Busby, P. E., G. Newcombe, R. Dirzo, and T. G. Whitham. 2014. Differentiating genetic and environmental drivers of plant-pathogen community interactions. Journal of Ecology 102:1300-1309.

Bushnell, W. R. 2002. The role of powdery mildew research in understanding host-parasite interaction: past, present and future. Pages 1-12 in R. R. Bélanger, W. R. Bushnell, A. J. Dik, and T. L. W. Carver, editors. The powdery mildews: a comprehensive treatise. APS Press, St. Paul, Minnesota, USA.

Calonnec, A., J. B. Burie, M. Langlais, S. Guyader, S. Saint-Jean, I. Sache, and B. Tivoli. 2013. Impacts of plant growth and architecture on pathogen processes and their consequences for epidemic behaviour. European Journal of Plant Pathology 135:479-497.

Christensen, R. H. B. 2015. ordinal: Regression Models for Ordinal Data. R package version 2015.6-28. http://www.cran.r-project. org/package $=$ ordinal/

Crawley, M. J. 2007. The R book. John Wiley and Sons Ltd, Chichester, UK.

Dwyer, G., J. S. Elkinton, and J. P. Buonaccorsi. 1997. Host heterogeneity in susceptibility and disease dynamics: tests of a mathematical model. American Naturalist 150:685-707.

Ekholm, A., T. Roslin, P. Pulkkinen, and A. J. M. Tack. 2017. Dispersal, host genotype and environment shape the spatial dynamics of a parasite in the wild. Ecology 98:2574-2584.

Estep, L. K., K. E. Sackett, and C. C. Mundt. 2014. Influential disease foci in epidemics and underlying mechanisms: a field experiment and simulations. Ecological Applications 24:1854-1862.

Gibson, G. J., C. A. Gilligan, and A. Kleczkowski. 1999. Predicting variability in biological control of a plant-pathogen system using stochastic models. Proceedings of the Royal Society B-Biological Sciences 266:1743-1753.

Gibson, A. K., J. Jokela, and C. M. Lively. 2016. Fine-scale spatial covariation between infection prevalence and susceptibility in a natural population. American Naturalist 188:1-14.

Glawe, D. A. 2008. The powdery mildews: a review of the world's most familiar (yet poorly known) plant pathogens. Annual Review of Phytopathology 46:27-51.

Hiemstra, P. H., E. J. Pebesma, C. J. W. Twenhofel, and G. B. M. Heuvelink. 2009. Real-time automatic interpolation of ambient gamma dose rates from the Dutch radioactivity monitoring network. Computers \& Geosciences 35:1711-1721.

Hijmans, R. J. 2015. raster: Geographic Data Analysis and Modeling. R package version 2.4-20. http://CRAN.R-project.org/packa $\mathrm{ge}=$ raster

Johnson, T. 1961. Man-guided evolution in plant rusts. Through his modification of the host plants of the cereal rusts, man is also modifying the rusts. Science 133:357-362.

Jones, L. B., J. Johnson, and J. G. Dickson. 1926. Wisconsin studies upon the relation of soil temperature to plant disease. Bull. Wis. Agric. Exp. Stn. Page 144.

Jousimo, J., A. J. M. Tack, O. Ovaskainen, T. Mononen, H. Susi, C. Tollenaere, and A.-L. Laine. 2014. Ecological and evolutionary 
effects of fragmentation on infectious disease dynamics. Science 344:1289-1293

King, K. C., and C. M. Lively. 2012. Does genetic diversity limit disease spread in natural host populations? Heredity 109:199-203.

Laine, A. L. 2004. Resistance variation within and among host populations in a plant-pathogen metapopulation: implications for regional pathogen dynamics. Journal of Ecology 92:990-1000.

Laine, A. L. 2007. Pathogen fitness components and genotypes differ in their sensitivity to nutrient and temperature variation in a wild plant-pathogen association. Journal of Evolutionary Biology 20:2371-2378.

Laine, A. L., and I. Hanski. 2006. Large-scale spatial dynamics of a specialist plant pathogen in a fragmented landscape. Journal of Ecology 94:217-226.

Laine, A. L., J. J. Burdon, P. N. Dodds, and P. H. Thrall. 2011. Spatial variation in disease resistance: from molecules to metapopulations. Journal of Ecology 99:96-112.

Lannou, C., S. Soubeyrand, L. Frezal, and J. Chadoeuf. 2008 Autoinfection in wheat leaf rust epidemics. New Phytologist 177:1001-1011

Mitchell, S. E., E. S. Rogers, T. J. Little, and A. F. Read. 2005. Hostparasite and genotype-by-environment interactions: temperature modifies potential for selection by a sterilizing pathogen. Evolution 59:70-80.

Mundt, C. C. 2002. Use of multiline cultivars and cultivar mixtures for disease management. Annual Review of Phytopathology 40:381-410.

Nicot, P. C., M. Bardin, and A. J. Dik. 2002. Basic methods for epidemiological studies of powdery mildews: culture and preservation of isolates, production and delivery of inoculum, and disease assessment. Pages 83-99 in R. R. Bélanger, W. R. Bushnell, A. J. Dik, and T. L. W. Carver, editors. The powdery mildews: a comprehensive treatise. APS Press, St. Paul, Minnesota, USA.

Ojanen, S. P., M. Nieminen, E. Meyke, J. Poyry, and I. Hanski. 2013. Long-term metapopulation study of the Glanville fritillary butterfly (Melitaea cinxia): survey methods, data management, and longterm population trends. Ecology and Evolution 3:3713-3737.

Ovaskainen, O., and A. L. Laine. 2006. Inferring evolutionary signals from ecological data in a plant-pathogen metapopulation. Ecology 87:880-891.

Papaï, J., L. Rimbaud, J. J. Burdon, J. Zhan, and P. H. Thrall. 2017. Differential impact of landscape-scale strategies for crop cultivar deployment on disease dynamics, resistance durability and long-term evolutionary control. Evolutionary Applications 11:705-717.

Parratt, S. R., E. Numminen, and A.-L. Laine. 2016. Infectious disease dynamics in heterogeneous landscapes. Annual Review of Ecology, Evolution and Systematics 47:283-306.

Penczykowski, R. M., B. C. P. Lemanski, R. D. Sieg, S. R. Hall, J. H. Ochs, J. Kubanek, and M. A. Duffy. 2014. Poor resource quality lowers transmission potential by changing foraging behaviour. Functional Ecology 28:1245-1255.

Penczykowski, R. M., A. L. Laine, and B. Koskella. 2016. Understanding the ecology and evolution of host-parasite interactions across scales. Evolutionary Applications 9:37-52.

R Core Team. 2015. R: a language and environment for statistical computing. R Foundation for Statistical Computing, Vienna, Austria.

Reiss, E. R., and L. E. Drinkwater. 2018. Cultivar mixtures: a metaanalysis of the effect of intraspecific diversity on crop yield. Ecological Applications 28:62-77.
Sagar, G. R., and J. L. Harper. 1964. Plantago major L., P. media L. and $P$. lanceolata L. Journal of Ecology 52:189-221.

Seppälä, O., A. Karvonen, M. Haataja, M. Kuosa, and J. Jokela. 2011. Food makes you a target: disentangling genetic, physiologi$\mathrm{cal}$, and behavioral effects determining susceptibility to infection. Evolution 65:1367-1375.

Shaw, M. W. 1995. Simulation of population expansion and spatial pattern when individual dispersal distributions do not decline exponentially with distance. Proceedings of the Royal Society B-Biological Sciences 259:243-248.

Smith, V. H., T. P. Jones, and M. S. Smith. 2005. Host nutrition and infectious disease: an ecological view. Frontiers in Ecology and the Environment 3:268-274.

Stevens, R. B. 1960. Cultural practices in disease control. Pages 357-429 in J. G. Horsfall and A. E. Dimond, editors. Plant pathology, an advanced treatise. Volume 3. Academic Press, New York, New York, USA.

Susi, H., and A. L. Laine. 2013. Pathogen life-history trade-offs revealed in allopatry. Evolution 67:3362-3370.

Susi, H., B. Barres, P. F. Vale, and A. L. Laine. 2015. Co-infection alters population dynamics of infectious disease. Nature Communications 6:5975.

Tack, A. J. M., and A. L. Laine. 2014. Ecological and evolutionary implications of spatial heterogeneity during the off-season for a wild plant pathogen. New Phytologist 202:297-308.

Tack, A. J. M., J. Hakala, T. Petäjä, M. Kulmala, and A.-L. Laine. 2014. Genotype and spatial structure shape pathogen dispersal and disease dynamics at small spatial scales. Ecology 95:703714.

Thompson, J. N., and J. J. Burdon. 1992. Gene-for-gene coevolution between plants and parasites. Nature 360:121-125.

Thorne, E. T., and E. S. Williams. 1988. Disease and endangered species: the black-footed ferret as a recent example. Conservation Biology 2:66-74.

Tollenaere, C., and A. L. Laine. 2013. Investigating the production of sexual resting structures in a plant pathogen reveals unexpected self-fertility and genotype-by-environment effects. Journal of Evolutionary Biology 26:1716-1726.

Truscott, J. E., and C. A. Gilligan. 2003. Response of a deterministic epidemiological system to a stochastically varying environment. Proceedings of the National Academy of Sciences of the United States of America 100:9067-9072.

Vale, P. F., M. Stjernman, and T. J. Little. 2008. Temperature-dependent costs of parasitism and maintenance of polymorphism under genotype-by-environment interactions. Journal of Evolutionary Biology 21:1418-1427.

Warren, R. J., and E. Mordecai. 2010. Soil moisture mediated interaction between Polygonatum biflorum and leaf spot disease. Plant Ecology 209:1-9.

Wolfe, M. S. 1985. The current status and prospects of multiline cultivars and variety mixtures for disease resistance. Annual Review of Phytopathology 23:251-273.

Wolinska, J., and K. C. King. 2009. Environment can alter selection in host-parasite interactions. Trends in Parasitology 25:236-244.

Zhan, J., P. H. Thrall, J. Papaix, L. Xie, and J. J. Burdon. 2015. Playing on a pathogen's weakness: using evolution to guide sustainable plant disease control strategies. Annual Review of Phytopathology 53:19-43.

Zhu, Y. Y., et al. 2000. Genetic diversity and disease control in rice. Nature 406:718-722. 